\title{
EFFECT OF DIFFERENT BASE MATERIALS ON THE, MICRO LEAKAGE AND FRACTURE RESISTANCE OF RECENT CERAMIC INLAY RESTORATIONS
}

\author{
Ola Barakat* and Noha Mohammed ${ }^{* *}$
}

\begin{abstract}
Purpose: The purpose of this study was to evaluate the effect of three different base materials (GIC, Chemfill rock, and SDR,) on microleakage, fracture resistance, and color stability of two ceramic inlays (E max and Vita suprinity).

Materials and methods: Sixty extracted premolars were prepared for Class II inlay MOD. For microleakage test thirty teeth divided into three groups $(n=10)$; group $(A)$ : restored with (GIC). Group (B): restored with Chemfill rock. Group (C): restored with SDR. After thermocycling the microleakage was evaluated by scores. For evaluation of fracture resistance and color stability, the remaining thirty teeth were divided into two groups(n=15) Group (1): The teeth restored with Emax inlay. Group (2): restored with Vita Suprinity inlay. Each group was subdivided into three subgroups(n=5); Subgroup (I): based with (GIC). Subgroup (II): based with Chemfill Rock. Subgroup (III): based with SDR.
\end{abstract}

Results: For microleakage test; SDR base had stage zero leakage, leakage was higher in GIC base. For fracture resistance test; there is statistically significant high mean of fracture resistance in SDR base. On the other hand there is no statistically significant difference as fracture resistance between E-max, and Vita Suprinity restoration. For color stability test; there is statistically significant difference high mean of color difference in SDR base with $\mathrm{p}$-value $<0.05$. On the other hand there is no statistically significant difference between E-max, and Vita Suprinity restoration.

Conclusion: SDR is the best base regarding microleakage, color stability and fracture resistance. While there is no significant difference between Emax and Vita Suprinity restorations. in color stability and fracture resistance.

* Associate Professor, Department of Operative Dentistry, Faculty of Oral and Dental Surgery, Misr University for Science and Technology. Egypt

** Lecture, Department of Fixed Prothodontic, Faculty of Oral and Dental Surgery, Misr University for Science and Technology. Egypt 


\section{INTRODUCTION}

Esthetic aspects of dental treatment are becoming increasingly important to patients, thus enhancing the demand for all ceramic restorations. Dental glass ceramics are used to replace missing or damaged tooth structure because of their high translucency, superior esthetics, biocompatibility, and low thermal diffusivity; however, being a brittle material, they display a high compressive and low tensile strength, which may cause restorations to fail in the oral environment. ${ }^{(1-7)}$

The polymerization shrinkage can result in gap formation between restorations of the cavity walls. Marginal gap formation contributes to micro leakage permitting the passage of oral fluids and bacteria from the oral cavity and become a source of postoperative sensitivity, pulpal inflammation and recurrent caries. ${ }^{(2-4)}$ To reduce these effects have been suggested, as a better option in Class II inlay restoration is using base. Glass-ionomer cement (GIC) is placed between the dentin cervical margins and inlay restoration. ${ }^{(5,6)}$ GICs have been shown to be less able to seal margins, can dissolve over time in the oral environment. ${ }^{(7-9)}$ The most recent attempt to reduce micro leakage uses new resin monomers with novel chemistries (low polymerization shrinkage) to compensate shrinkage stress. SDR ${ }^{\mathrm{TM}}$ (Dentsply Caulk) is designed to reduce micro leakage by increasing flow with a unique chemistry that slows the rate of polymerization to reduce shrinkage stress This composite resin is used as a dentin replacement material and polymerized in 4-mm increments. ${ }^{(10-}$ 13) On another hand, the manufacturer of a recently launched GIC (ChemFill Rock, Dentsply) followed a different approach to enhance material's stability, claiming that surface protection in the form of resin coating is irrelevant for product's performance. An enhanced setting reaction in the new GIC is supposed, due to the zinc content as part of its glass particles, leading thus to higher strength, by similar working time and application comfort as regular GICs. ${ }^{(14,15)}$
Lithium disilicate ceramic (such as IPS E.max $\mathrm{CAD}$, manufactured by Ivoclear Vivadent) is considered one of the more esthetic ceramics due to the glass matrix embedded with needle-like lithium disilicate crystals that results in reduction of internal scattering of the light as it passes through the material. ${ }^{(16)}$ There are also other optical properties for better mimicking of adjacent natural teeth, which include the chameleon effect. Since the fracture resistance of lithium disilicate glass ceramic is in general less than zirconia, Recently introduced ceramic Vita Suprinity (Vita, Zahnfabrik, Bad Sackingen, Germany) is a lithium silicate ceramic enriched with zirconia (approx..10\%). This new glass ceramic features a special fine grained and homogenous structure, which guarantees excellent material quality, consistent high load capacity and excellent translucency. The null hypothesis of the study was that there is no significant difference in micro leakage of the different evaluated restorative techniques evaluated.

\section{MATERIALS AND METHODS}

Sixty extracted non-carious intact human permanent maxillary premolars were used in this study. They stored in distilled water after cleaning. The tooth dimensions were approximately closed to each other (a bucco-lingual width), (a mesio-distal width) and (an occluso-gingival height). They were measured using caliper. The roots of each tooth were embedded in plastic tubes filled with acrylic resin (Formatray, Kerr, Orange, CA, USA) at a level approximately one $\mathrm{mm}$ below the cemento enamel junction. The long axis of the tooth was perpendicular to the horizontal plane. A Class II inlay MOD cavity was prepared on each tooth using a taper diamond bur (Inlay set No 8113R, Intensive SA, Swiss Dental Products, Lugano-Grancia, Switzerland), with a $6^{\circ}$ angle of inclination. A high-speed handpiece was attached to the paralleling device; the long axis of the diamond bur was positioned parallel to the long axis of the tooth. Water spray was used during the preparation procedure. Following five preparations, a new bur was used. 
The cavity dimensions are: the cavity depth, 1.5 $\pm 0.1 \mathrm{~mm}$, was measured from the deepest point of the main fissure to the level of the pulpal floor. The bucco-lingual width was $3.0 \pm 0.1$, the width of the gingival wall was $1.2 \pm 0.1$ and the height of the axial wall was $2.0 \pm 0.1 \mathrm{~mm}$. All internal line angles and point angles were rounded. All cavity margins were placed within enamel without bevel. The preparation was finished with a fine diamond bur (Inlay set No 3113R, Intensive SA, Swiss Dental Products).

\section{Microleakage test:}

Thirty prepared teeth were divided into three groups:

Group (A): The prepared cavity was restored completely with glass ionomer (GIC) base material.

Group (B): The prepared cavity was restored completely with Chemfill rock base material(CF).

Group (C): The prepared cavity was restored completely with SDR base material.

These specimens were subjected to thermo cycling included an alternative exposure of specimens to temperatures of $5^{\circ}$ and $55^{\circ} \mathrm{C}$ for 30 seconds. The procedure was repeated 50000 times.

The specimens were then soaked in an aqueous solution of $2 \%$ methylene blue dye for 24 hours at room temperature. Following dye exposure, the teeth were rinsed thoroughly with an air/water spray for 30 seconds and dried.

The roots of specimens were cut until $2 \mathrm{~mm}$ below cemento enamel junction. The specimens were embedded in blocks of acrylic resin. Each embedded specimens was sectioned bucco-lingually with an Isomet slow-speed, water-cooled diamond saw, producing two sections (replications) from each tooth.

Micro leakage was evaluated using stereomicroscope at magnification 20X. Extent of dye penetration was checked at the tooth/base interface. The extent of dye penetration was evaluated using the following scale:
Scale 0: No penetration.

Scale 1: Cervical wall penetration.

Scale 2: Axial wall penetration.

Scale 3: Pulpal floor penetration.

\section{Inlay fabrication:}

Thirty teeth were divided into two groups (15 each) the inlay were constructed using CAD/CAM milling machine, Cerec inlab system* Inlab 4, Sirona dental systems GMBL+, Germany.

Group (1): The teeth restored with E max ceramic restoration.

Group (2): The teeth restored with Vita Suprinity ceramic restoration.

Each group was divided into three subgroups (5 each)

Subgroup I: cavities that based on a $1 \mathrm{~mm}$ GIC base.

Subgroup II: cavities that based on a $1 \mathrm{~mm}$ SDR base.

Subgroup III: cavities that based on a $1 \mathrm{~mm} \mathrm{CF}$ base.

The shade of the selected teeth, the bases and the ceramic restorations used was A3

After restoration fabrication the Color stability test was done to the inlay restorations before cementation:

Color stability test: A spectrophotometer was used for the reflectance measurements of the inlay restorations before cementation.

\section{Inlays cementation}

Rely $\mathrm{X}^{\mathrm{TM}}$ Ultimate used in a self-etch mode according to the manufacturer's instructions and recommendations, the prepared teeth were cleaned with water and then dried with cotton pellets. 
- The Cement capsule was placed into the activator holder and the activating lever was pressed all the way down and held for 3 seconds. This is important to ensure that all liquid in the sachet is squeezed into the mixing chamber.

- The capsule was immediately inserted $0020 \mathrm{dcx}$ in a mixer.

- RelyX $^{\mathrm{Tm}}$ Ultimate Cement was mixed for 8 seconds.

- The capsule was immediately inserted into the applicator and was applied into the fitting surface of the inlays.

- Each inlay was seated on its corresponding cavity under static pressure of $3 \mathrm{Kg}$ for 5 minutes using a specially designed cementation device

- Excess material was removed from the margin using dental probe when the cement has reached the rubber-like consistency.

- The cemented inlays were light cured for 40 seconds from all sides of restoration.

1- Color stability test: A spectrophotometer was used for the reflectance measurements of the inlay restorations before and after cementation. This in order to evaluate the effect of base on the shade color of restoration. The mean color difference values $(\Delta \mathrm{E})$ between each group before and after cementation were calculated using the following CIE $\mathrm{L}^{*}, \mathrm{a}^{*}, \mathrm{~b}^{*}$ color difference formula.

$$
\Delta E=\left(\Delta L^{2}+\Delta a^{2}+\Delta b^{2}\right)^{1 / 2}
$$

$\mathrm{L}^{*}$ in color space represents the luminance of the color (or Value) on a numerical scale from 0 (black) to 100 (white). The color coordinates $\mathrm{a}^{*}$ and $b^{*}$ represent a position between red $\left(+a^{*}\right)$, and green $\left(-\mathrm{a}^{*}\right)$, and between yellow $\left(+\mathrm{b}^{*}\right)$ and blue $(-$ $\left.b^{*}\right)$. The values of $L^{*}, a^{*}, b^{*}$ color coordinates are known as CIELAB values were determined from 3 measurements at the center of the restoration.

\section{Fracture resistance test and its type.}

Five inlay restorations of each group were subjected for fracture testing using computer controlled materials testing machine (Model LRXplus; Lloyd Instruments Ltd., Fareham,) All samples were individually mounted vertically with a load cell of $5 \mathrm{kN}$. Data were recorded using computer software (Nexygen-MT; Lloyd Instruments).

Restoration was secured to the lower fixed compartment of the machine by tightening screws. Load was applied with a custom made load applicator (A steel rod ball $5.8 \mathrm{~mm}$ diameter) attached to the upper movable compartment of the machine. A tin foil was placed between the loading tip and the occlusal surface of specimens to achieve an even stress distribution and to minimize the transmission of local force peaks. Specimens were statically compression loaded until fracture at a crosshead speed of $1 \mathrm{~mm} / \mathrm{min}$.

Failure manifested by the first sound crack and confirmed by sudden drop along the load-deflection curves was recorded with the computer software.

The type of fracture was evaluated according to the following classification:

Type I: fractures involving a small portion of the coronal tooth surface; adhesive.

Type II: fractures involving a small portion of the coronal tooth structure and restoration cohesive failure;

Type III: fractures involving the dental structure, restoration cohesive, and/or adhesive failure with root involvement that can be restored with periodontal surgery.

Type IV: severe fractures involving the root and crown and resulting in tooth extraction.

\section{RESULTS}

- Data were collected and coded to facilitate data manipulation and double entered into Microsoft Access and data analysis was performed using 
Statistical Package of Social Science (SPSS) software version 18 in windows 7.

- Simple descriptive analysis in the form of numbers and percentages for qualitative data, and arithmetic means as central tendency measurement, standard deviations as measure of dispersion for quantitative parametric data.

- Quantitative data included in the study was first tested for normality by One-Sample Kolmogorov-Smirnov test in each study group then inferential statistic tests were selected.

\section{For quantitative parametric data}

- In-depended student t-Test used to compare measures of two independent groups of quantitative data

- One way ANOVA test in comparing more than two independent groups of quantitative data.

\section{For quantitative non parametric data}

\section{Non Paired variables}

- kruskal wallis test used in comparing more than two independent groups.

- Mann-whitney test in comparing two independent groups.

\section{For qualitative data}

- Chi square test to compare two of more than two qualitative groups.

- The $\mathbf{P}$-value $\leq \mathbf{0 . 0 5}$ was considered the cut-off value for significance.

\section{Microleakage result}

Table (1) figure (1) illustrates that there is high statistically significant difference between the 3 bases. SDR base had stage zero leakage, both $\mathrm{CF}$ and GIC base finally leakage was higher in GIC base with p-value $<0.05$.

TABLE (1): Comparisons of leakage degrees in different study groups.

\begin{tabular}{|c|c|c|c|c|}
\hline \multirow{3}{*}{ Leakage } & \multicolumn{3}{|c|}{ Study groups } & \multirow{3}{*}{ p-value } \\
\hline & $\begin{array}{c}\text { GI } \\
(\mathrm{n}=10)\end{array}$ & $\begin{array}{c}\mathbf{C F} \\
(\mathrm{n}=10)\end{array}$ & $\begin{array}{c}\text { SDR } \\
(\mathrm{n}=10)\end{array}$ & \\
\hline & No. $(\%)$ & No. $(\%)$ & No. $(\%)$ & \\
\hline Stage 0 & $0(0 \%)$ & $0(0 \%)$ & $5(50 \%)$ & \multirow{4}{*}{$0.004 *$} \\
\hline Stage 1 & $0(0 \%)$ & $2(20 \%)$ & $0(0 \%)$ & \\
\hline Stage 2 & $3(30 \%)$ & $2(20 \%)$ & $4(40 \%)$ & \\
\hline Stage 3 & $7(70 \%)$ & $6(60 \%)$ & $1(10 \%)$ & \\
\hline
\end{tabular}

*statistical significance difference with p-value $<0.05$

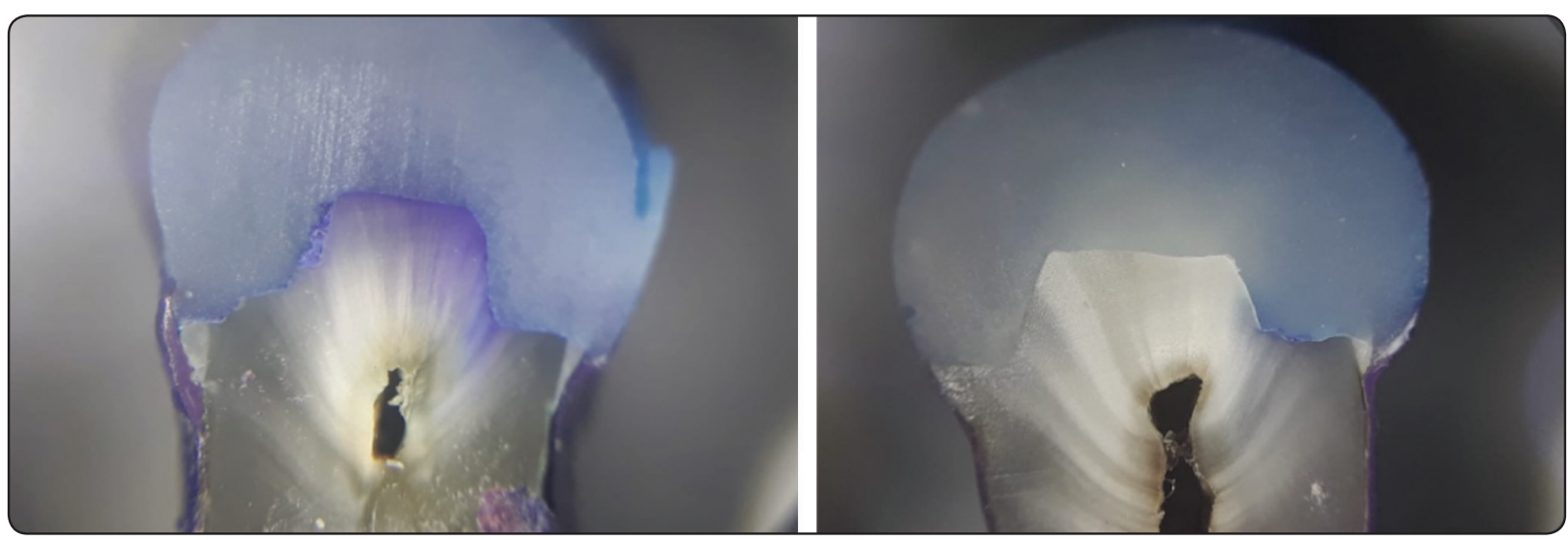

(a) Grade 4 leakage in GIC(b)Grade 1 leakage in SDR

Fig. (1) Illustrate grades of leakage (a) grade 4 for GIC and (b) grade 1 for SDR 


\section{Color result}

Table (2) Figure (2a, 2b) illustrates that there is statistically significant difference high mean of color difference in SDR base with p-value $<0.05$. On the other hand there is no statistically significant difference with $\mathrm{p}$-value $>0.05$ as regards color difference between E-max, and Vita suprinity restoration Figure (3).

TABLE (2): Comparisons of color difference between different bases and restoration.

\begin{tabular}{|c|c|c|}
\hline \multirow{2}{*}{ Variables } & Color difference & \multirow{2}{*}{ p-value } \\
\hline & Mean \pm SD & \\
\hline \multicolumn{3}{|l|}{ Bases type } \\
\hline GI & $1.7 \pm 3.3$ & \multirow{3}{*}{$0.04 *$} \\
\hline $\mathrm{CF}$ & $2.9 \pm 1.1$ & \\
\hline SDR & $3.5 \pm 0.65$ & \\
\hline \multicolumn{3}{|l|}{ Restorations } \\
\hline E-Max & $2.8 \pm 0.72$ & \multirow{2}{*}{0.9} \\
\hline Vita suprinity & $2.9 \pm 3.1$ & \\
\hline
\end{tabular}

*statistical significance difference with p-value $<0.05$
Table (3) illustrates that there is statistically significant high mean of color difference in Vita suprinity restoration versus E-max type on both $\mathrm{CF}$, and SDR base with $\mathrm{p}$-value $<0.05$. On the other hand there is no statistically significant difference with p-value $>0.05$ as regards color difference between E-max, and vita suprinity among GIC base.

Among E-max cases there is statistical significant high mean of color difference on SDR base, but high fracture resistance on $\mathrm{CF}$ base.

Among Vita suprinity cases there is statistical significant high mean of color difference and fracture resistance on SDR base, Figure (3).

TABLE (3): Comparisons of color difference between different restoration and bases.

\begin{tabular}{|c|c|c|c|}
\hline \multirow{2}{*}{$\begin{array}{c}\text { Color } \\
\text { difference }\end{array}$} & $\begin{array}{c}\text { E-max } \\
(\mathrm{n}=5)\end{array}$ & $\begin{array}{c}\text { Vita Suprinity } \\
(\mathrm{n}=5)\end{array}$ & \multirow{2}{*}{ p-value ${ }^{\mathrm{B}}$} \\
\cline { 2 - 4 } & Mean \pm SD & Mean \pm SD & \\
\hline GI & $13.11 \pm 0.26$ & $10.19 \pm 4.3$ & 0.17 \\
\hline CF & $11.85 \pm 0.20$ & $\mathbf{1 3 . 8 5} \pm \mathbf{0 . 1 9}$ & $<_{\mathbf{0 . 0 0 1}}^{*}$ \\
\hline SDR & $\mathbf{1 3 . 4 2} \pm \mathbf{0 . 1 4}$ & $\mathbf{1 4 . 5 7 \pm 0 . 2 9}$ & $<\mathbf{0 . 0 0 1}^{*}$ \\
\hline p-value $^{\text {A }}$ & $<\mathbf{0 . 0 0 1 *}$ & $\mathbf{0 . 0 3}$ & \\
\hline
\end{tabular}

*statistical significance difference with p-value $<0.05$ p-value ${ }^{A}$ : significance between 3 bases p-value ${ }^{B}$ : significance between 2 restorations

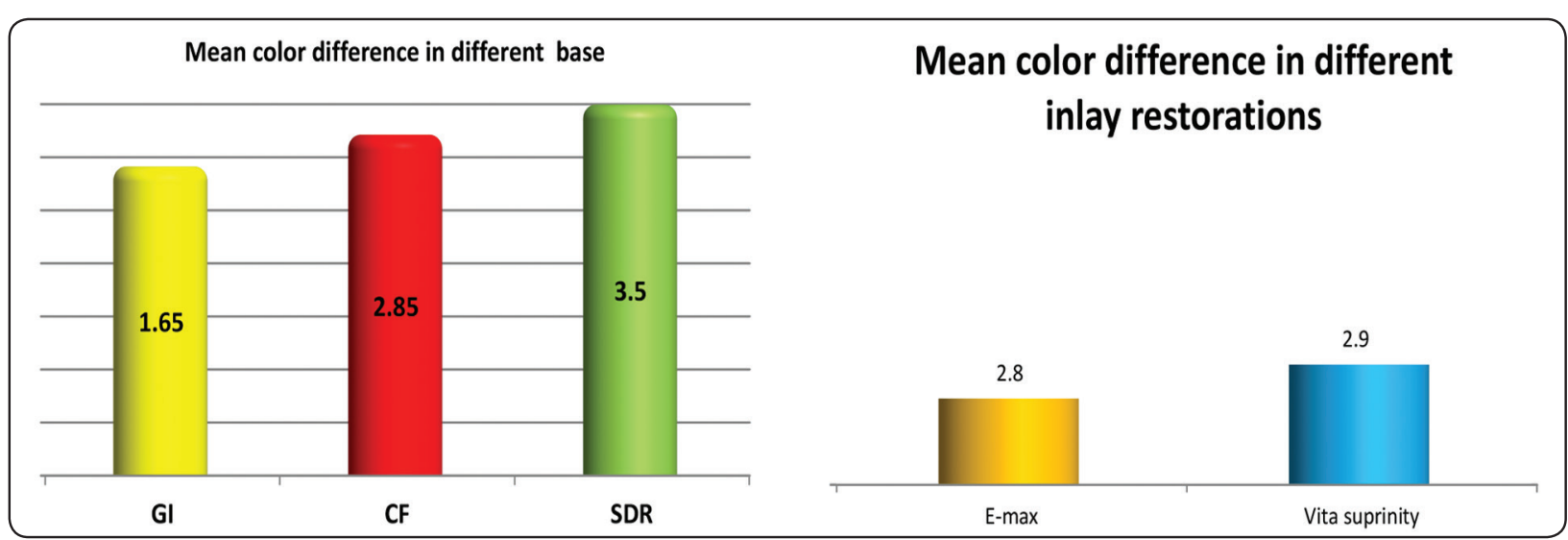

Fig. (1)

Fig. (2) 


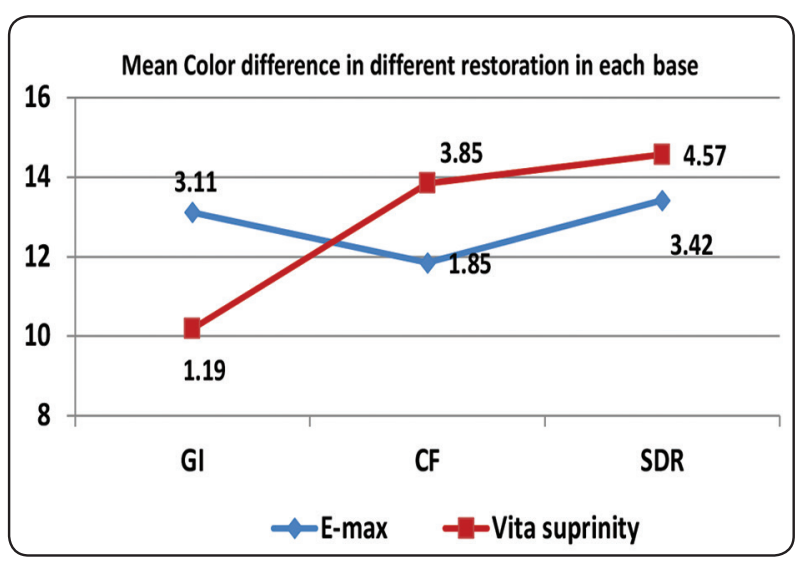

Fig. (3)

\section{Fracture resistance result}

Table (4) illustrates that there is statistically significant high mean of fracture resistance in SDR base; with p-value $<0.05$, Figure (4a). On the other hand there is no statistically significant difference with p-value $>0.05$ as regards fracture resistance between E-max, and Vita suprinity restoration, Figure (4b).

Table (5) illustrates that there is statistically significant high mean of fracture resistance in Vita suprinity restoration versus E-max type on both GIC, and SDR base and high mean fracture resistance in E-max restoration on CF base with p-value $<0.05$.
Among E-max cases there is statistical significant high mean of fracture resistance on CF base. Among Vita suprinity cases there is statistical significant high mean of fracture resistance on SDR base, Figure (5).

TABLE (4): Comparisons of fracture resistance between different bases and restoration.

\begin{tabular}{|c|c|c|}
\hline \multirow{2}{*}{ Variables } & Fracture resistance & \multirow{2}{*}{ p-value } \\
\hline & Mean \pm SD & \\
\hline \multicolumn{3}{|c|}{ Bases type } \\
\hline GI & $464.5 \pm 21.2$ & \multirow{3}{*}{$<0.001 *$} \\
\hline $\mathrm{CF}$ & $639.4 \pm 41.5$ & \\
\hline SDR & $707.5 \pm 111.1$ & \\
\hline \multicolumn{3}{|c|}{ Restoration } \\
\hline E-Max & $575.9 \pm 99.3$ & \multirow{2}{*}{0.2} \\
\hline Vita suprinity & $631.7 \pm 142.3$ & \\
\hline
\end{tabular}

*statistical significance difference with p-value $<0.05$

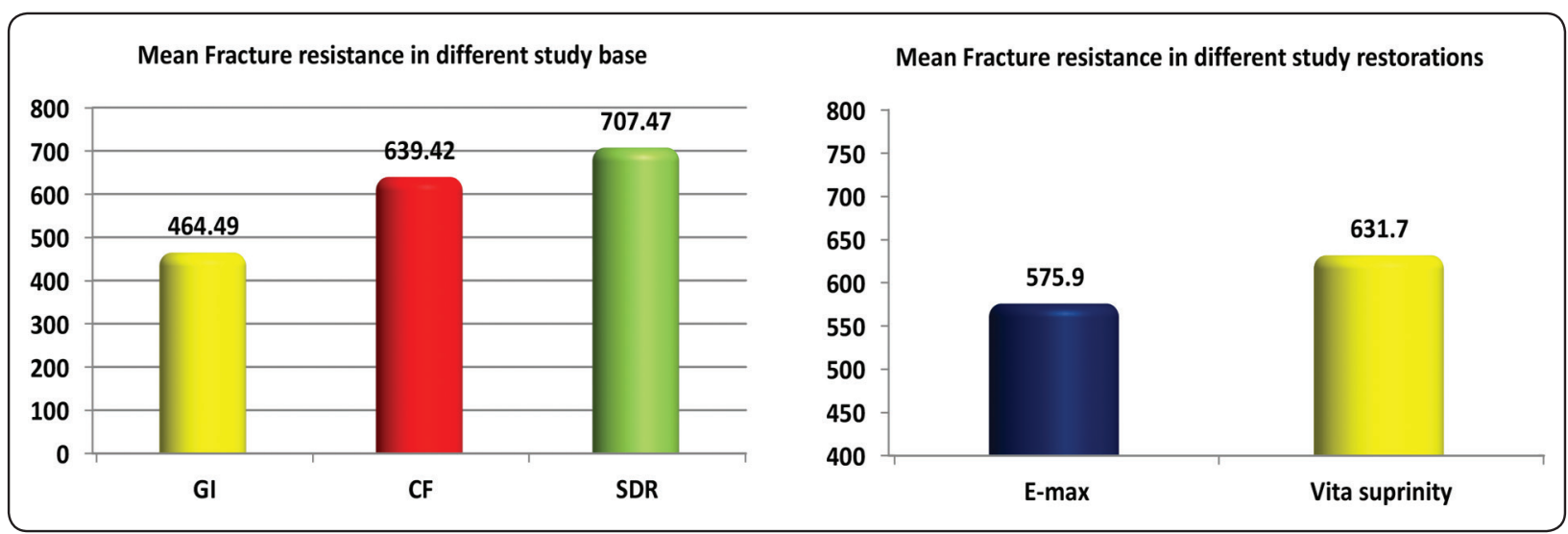

Fig. (4a)

Fig. (4b) 
TABLE (5): Comparisons of fracture resistant between different restoration and bases.

\begin{tabular}{|c|c|c|c|}
\hline \multirow{2}{*}{$\begin{array}{l}\text { Fraction } \\
\text { resistance }\end{array}$} & $\begin{array}{c}\text { E-max } \\
(\mathrm{n}=5)\end{array}$ & $\begin{array}{c}\text { Vita suprinity } \\
\qquad(\mathrm{n}=5)\end{array}$ & \multirow[t]{2}{*}{ p-value ${ }^{\mathrm{B}}$} \\
\hline & Mean \pm SD & Mean \pm SD & \\
\hline GI & $448.35 \pm 10.9$ & $480.63 \pm 15.6$ & $0.005 *$ \\
\hline $\mathrm{CF}$ & $674.71 \pm 20.6$ & $604.14 \pm 18.3$ & $<0.001 *$ \\
\hline SDR & $604.48 \pm 20.7$ & $810.45 \pm 29.2$ & $<0.001 *$ \\
\hline p-value ${ }^{A}$ & $<0.001 *$ & $<0.001 *$ & \\
\hline
\end{tabular}

*statistical significance difference with $p$-value $<0.05$

-value A: significance between 3 bases

p-value B: significance between 2 restorations

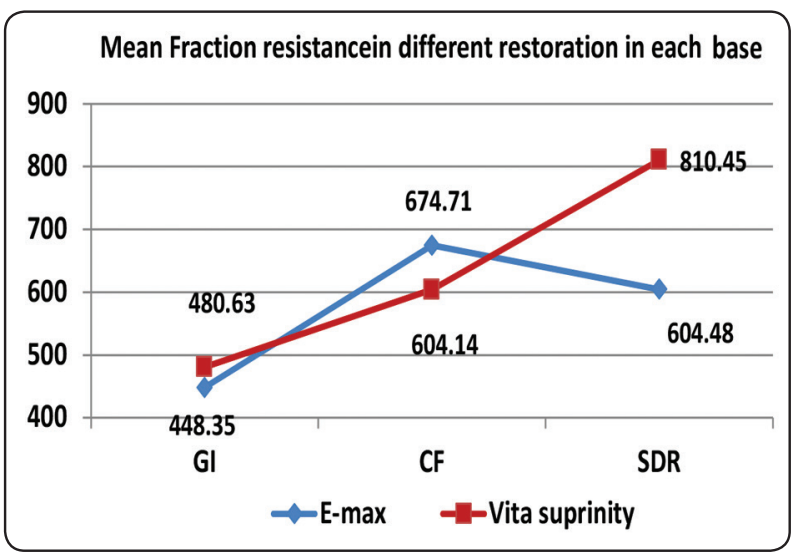

Fig. (5)

\section{Fracture type determination:}

SDR base: the restorations based with SDR base showed Type III fractures that involved the dental structure, restoration cohesive, and/or adhesive failure with root involvement.

CF base: the restorations based with $\mathrm{CF}$ base showed Type II fractures that involved a small portion of the coronal tooth structure and restoration cohesive failure.

GIC base: the restorations based with GIC base showed_Type I fractures involved a small portion of the coronal tooth surface.

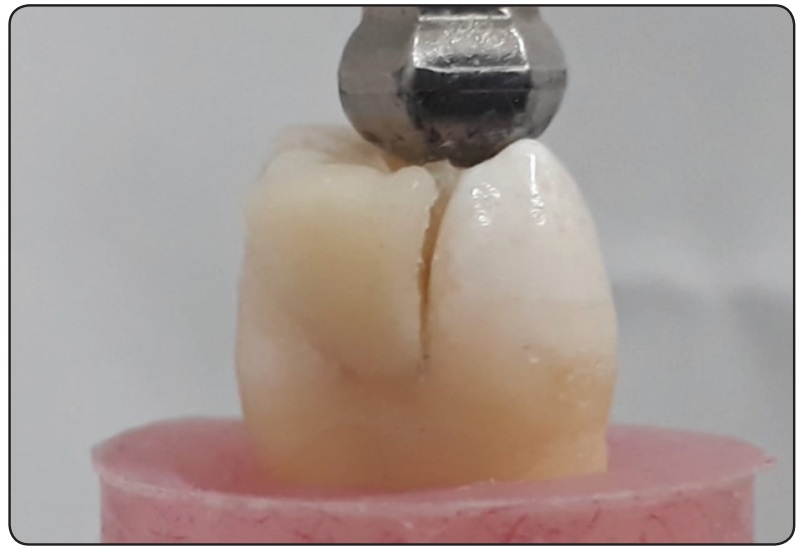

Fig. (7) Fracture resistance test

\section{DISCUSSION}

During the past decade, the use of all-ceramic inlay/onlay restorations has expanded due to improvements in dental adhesives and resin cements, but in deep cavities the use of a base under ceramic inlays are recommended. The effect of the base materials under Class II ceramic inlays luted with a resin composite should be evaluated. ${ }^{(17)}$

Microleakage measurement provides an assessment of the marginal adaptation by evaluating dye penetration between the tested material and the tooth structure. This method is the most commonly used for assessing the sealing efficiency of a restorative system and the longevity of a restoration thus, it is essential to evaluate it. In the present in vitro study, we compared the marginal microleakage of three base materials: A new improved GICs (ChemFil ${ }^{\mathrm{TM}}$ Rock), SDR and conventional GIC in class II restoration.

Microleakage is governed by marginal adaptation of the restorative material to the tooth and is influenced by polymerization shrinkage and coefficient of thermal expansion. Ideally, filling materials and dental tissues should have identical coefficients of thermal expansion in order to limit leakage at the margins of the restorations. ${ }^{(18)}$

To simulate thermal stresses on the tooth restoration interface, microleakage studies usually employ thermocycling of different regimens. 
A consolidated view indicates that various material properties (e.g., thermal expansion, elasticity) seem to play a role in modifying the marginal sealing ability; non material-related factors (e.g., cavity configuration, application technique, and curing method) have to be taken into account as well. ${ }^{(19)}$

In this study, the dynamic environment of the oral cavity was simulated by exposing the three bases materials to thermal changes via thermocycling. Thermal cycles ranging between 50000 times were used.

For the qualitative measurements we measured the degree of microleakage on a 0-3 scale using the same images.

Dye penetration test was used to assess marginal leakage in this study. It is a widely accepted and generally preferred method because it is readily available, cheap, and non-toxic. In addition, the most effective dye for revealing microleakage $2 \%$ methylene blue dye was used in this study.

The results of this in vitro study revealed that there is high statistically significant difference between the 3 bases materials in microleakage test. SDR base had stage zero leakage, both chemfill and GIC base leakage was higher in GIC base with pvalue $<0.05$.

In this study the SDR group (SDR flow E modulus $=5.5 \mathrm{GPa}$ ) presented a better adaptation than the chemfill and GI groups. These findings are in accordance with those reported by (C Shahidi et al 2017)(20)

The low viscosity of SDR system facilitate plastic flow during the early phases of polymerization could be responsible for the better adaptation exhibited by this restorative materials (Scotti et al 2014) this flowable resin serve as a stress-absorbing layer during polymerization shrinkage and act by reducing the effect of the C-factor. Another study showed similar level of micro leakage of SDR(Moorthy A et al 2012) ${ }^{(22)}$.
The result of this study was disagreement with (Sertac Peker,et al 2016) ${ }^{(23)}$ as they found that; glass ionomer cements adhere to dental structures through chemical adhesion when the carboxylic groups of cement bind to tooth calcium ions. Glass ionomers also possess coefficient of thermal expansion close to that of the tooth structures and present low setting shrinkage. ${ }^{(24)}$ This provides eventually good marginal sealing, minimal microleakage at the restoration/tooth interface and thereby high retention rate. ${ }^{(25)}$

Chemfill rock and conventional glass ionomer showed less micro leakage than SDR

But there was no significant difference. Interestingly, chemfill glass ionomer cements presented less micro leakage than conventional glass ionomer .In the current study; the manufacturer's instructions were followed strictly. Additional cavity conditioner application was not recommended for chemfill glass ionomer. Cavity conditioner provided for this product is composed of aqueous polyacrylic acid with aluminum chloride. While the aluminum chloride acts as a wetting promoter, polyacrylic acid provides the carboxyl group for hydrogen bonding, which is then displaced by the stronger interaction of polar and ionic attraction from the glass ionomer setting reaction. (26) Apparently, pre-treatment of prepared cavities with the conditioner in the conventional glass ionomer system significantly improved the marginal adaptation compared to the chemfill

While, the addition of zinc oxide particles is the essential modification of chemfill,which also contains a high-molecular-weight acrylic acid polymer. The manufacturer maintains that inclusion of zinc oxide enhances the setting reaction and increases strength, while retaining similar methods of clinical application and working time as compared with regular GICs. ${ }^{(27)}$

In this study, the inlays were fabricated and bonded to the extracted human teeth to mimic clinical conditions. The inlays supported by dentin and 
SDR failed catastrophically; whereas those supported by GI had a non-linear load-displacement curve. This indicated that plastic deformation occurred in the latter group before failure occurred. Failure of ceramic restorations usually results from tensile stresses, because ceramic has a tensile strength much lower than its compressive strength. ${ }^{(28)}$ Occlusal loading causes flexural deformation of the restoration, resulting in tensile stresses occurring on the internal surface, which is the origin of the fracture. With rigid support, flexion of the restoration and, therefore, plastic deformation, is reduced. The use of base materials with sufficiently high elastic moduli, for example SDR, can provide sufficient support to reduce tensile stress on the internal surface of ceramic inlays. This also gives rise to high resistance to occlusal load of the restoration. On the other hand, the use of base materials with low elastic moduli, for example, GIC, allows for deflection of the restorations above them, thus producing a large amount of tensile stresses in the restoration, which leads to restoration fracture.

Scherrer and de Rijk ${ }^{(29)}$ reported the effect of the elastic modulus of supporting structure on the fracture load of all-ceramic crowns. They showed that fracture load increased when the elastic modulus of a core material or the supporting structure increased. Lee and Wilson ${ }^{(30)}$ also found the effect of different elastic moduli of cores on the fracture resistance of aluminous porcelain jacket crowns. They recommended that high elastic modulus materials be used for the core build-up of all ceramic crowns. Farah, Hood and Craig ${ }^{(31)}$ reported that a base material should have a modulus of elasticity as high as possible to support a restoration from intermittent forces during mastication.

A new dental ceramic material is developed, Vita Suprinity (Vita Zahnfabrik) is a glass ceramic which enriched with zirconia (approx.10 \% by weight), resulting in zirconia reinforced lithium silicate ceramic (ZLS). This glass ceramic featured a special fine-grained and homogeneous structure, which was claimed by the manufacturer to provide excellent physical qualities, consistent high load capacity, long-term reliability and excellent esthetic properties ${ }^{(32)}$

CIELAB (Commission Internationale de I'Eclairage) color coordinates system is a very useful mode, providing information about location of object color in a uniform 3 dimensional color space as documented by Gupta et al. ${ }^{(33)}$ to determine color difference, it is necessary to compute and record the difference in all three color space Values, $L^{*}, a^{*}, b^{*}$. In the present study there were no statistically significant difference with $p$-value $>0.05$ as regards color difference between E-max, and Vita suprinity restoration. This was agreed with Marwa Tannir et al (34) study, they compared the color stability and fracture strength of two hybrid ceramic (Suprinity and Enamic) veneers versus Lithium Disilicate ceramic veneers. They also found that; there were no significant differences between the groups; Suprinity exhibited the best color stability values. This may attributed to the homogeneous, fine crystalline structure of Suprinity versus needle-shaped crystals in the case of lithium disilicate ceramic.

Regarding fracture resistance testing, this study revealed that; there was statistically non significant high mean of fracture resistance in Vita suprinity restorations versus E-max restorations. That may attributed to, the incorporated zirconia grains act as nuclei for crystallization producing a greater number of smaller crystallites $(0.5-1 \mu \mathrm{m})$ rather than the fewer large crystallites $(1.5 \mu \mathrm{m})$ that are present in the IPS E.max CAD. Meanwhile, the incorporated zirconia will increase the fracture toughness via the stress-induced toughening mechanism that prevents crack propagation ${ }^{(35)}$.

The above finding is in agreement with Preis et al. (36) and Franz et al. ${ }^{(37)}$ who compared the fracture strength of crowns fabricated from zirconiareinforced lithium silicate and lithium disilicate and found that crowns fabricated zirconia-reinforced lithium silicate showed higher mean value of fracture strength than those fabricated from lithium disilicate. 
However, the above finding disagrees with the findings of Sieper et al. ${ }^{(38)}$ tested the fracture strength of crowns fabricated from lithium disilicate, zirconia-reinforced lithium silicate and other all-ceramic materials and found that the fracture strength of all-ceramic crowns fabricated from lithium disilicate was higher than that for zirconiareinforced lithium silicate crowns.

From the fracture surface analysis, the failure origins of most specimens were at the glazed surfaces. For zirconia-reinforced lithium silicate glass-ceramics, the glaze layer of these materials was more homogeneous and resulted in a very small failure origin when compared with a lithiumdisilicate-based glass-ceramic. A large defect in the glaze layer of a lithium-disilicate-based glassceramic could result from the heterogeneity of material compositions or porosity created during glazing material application. This large defect in the glaze layer was also observed in leucite-based glassceramic (IPS Empress CAD). This large defect in the glaze layer might be the reason for the lower strength for dental glass-ceramic materials.

\section{CONCLUSION}

SDR is the best base regarding micro leakage, color stability and fracture resistance. While there is no significant difference between Emax and Vita Suprinity restorations in color stability and fracture resistance.

\section{REFERANCES}

1. Yip KH, Poon BK, Chu FC, Poon EC, Kong FY, Smales RJ. Clinical evaluation of packable and conventional hybrid resin-based composites for posterior restorations in permanent teeth: Results at 12 months. J Am Dent Assoc. 2003;134:1581-9.

2. Attar N, Korkmaz Y. Effect of two light-emitting diode (LED) and one halogen curing light on the microleakage of Class V flowable composite restorations. J Contemp Dent Pract. 2007;8:80-8.

3. Pashley DH. Clinical considerations of microleakage. J Endod. 1990;16:70-7.
4. Sadeghi M. The effect of fluid composite as gingival layer on microleakage of class II composite restorations. Dent. Res J. 2007;4:40-7.

5. Besnault C, Attal JP. Simulated oral environment and microleakage of class II resin-based composite and sandwich restorations. Am J Dent. 2003;16:186-90.

6. Loguercio AD, Reis A, Mazzocco KC, Dias AL, Busato AL, Singer JM. Microleakage in class II composite resin restorations: total bonding and open sandwich technique. $\mathrm{J}$ Adhes Dent. 2002;4:137-44.

7. Holtan JR, Nystrom GP, Douglas WH, Phelps RA. Microleakage and marginal placement of a glass-ionomer liner. Quintessence Int. 1990;21:117-22.

8. Stockton LW, Tsang ST. Microleakage of Class II posterior restorations with gingival margins entirely within dentin. J Can Dent Assoc. 2007;73:255.

9. Welbury RR, Murray JJ. A clinical trial of the glassionomer cement-composite resin "sandwich" technique in Class II cavities in permanent premolar and molar teeth. Quintessence Int. 1990;21:507-12.

10. Neme AM, Maxson BB, Pink FE, Aksu MN. Microleakage of Class II packable resin composites lined with flowables: An in vitro study. Oper Dent. 2002;27:600-5.

11. Fabianelli A, Sgarra A, Goracci C, Cantoro A, Pollington S. Microleakage in class II restorations: open vs closed centripetal build-up technique. Oper Dent. 2010;35:308-13.

12. Sadeghi M. Influence of flowable materials on microleakage of nanofilled and hybrid Class II composite restorations with LED and QTH LCUs. Indian J Dent Res. 2009;20:159-63.

13. Ozgunaltay G, Gorucu J. Fracture resistance of class II packable composite restorations with and without flowable liners. J Oral Rehabil. 2005;32:111-5.

14. Beznos C. Microleakage at the cervical margin of composite Class II cavities with different restorative techniques. Oper Dent. 2001;26:60-9.

15. Burgess J, Cakir D. Comparative properties of low-shrinkage composite resins. Compend ContinEduc Dent. 2010;31 Spec No 2:10-5.

16- Holand W, Schweiger M, Frank M and Rheinberger V. A comparison of microstructure and properties of the IPS Empress and the IPS Empress Glass ceramics. J Biomed Mater Res. 2000; 53: 297 - 303. 
17- S. Banditmahakun, W. Kuphasuk, W. Kanchanavasita, and C. Kuphasuk .The Effect of Base Materials with Different Elastic Moduli on the Fracture Loads of Machinable Ceramic Inlays. Operative Dentistry: February 2006, Vol. 31, No. 2, pp. 180-187.

18-.Quo BC, Drummond JL, Koeber A, Fadavi S, Punwani I. Glass ionomer microleakage from preparations by an Er/YAG laser or a high-speed handpiece. J Dent 2002;30:141-146.

19- Krifka S, Federlin M, Hiller KA, Schmalz G. Microleakage of silorane- and methacrylate-based class $\mathrm{V}$ composite restorations. Clin Oral Investig 2012; 16(4):1117-24.

20- Shahidi C, Krejci I, Dietschi D. In Vitro Evaluation of Marginal Adaptation of Direct Class II Composite Restorations Made of Different "Low-Shrinkage" Systems Oper Dent. 2017;42(3):273-283.

21- Scotti N, Comba A, Gambino A, et al. Microleakage at enamel and dentin margins with a bulk fills flowable resin. Eur J Dent. 2014;8(1):1-8.

22- Moorthy A, Hogg CH, Dowling AH, Grufferty BF, Benetti AR, Fleming GJ: Cuspal deflection and microleakage in premolar teeth restored with bulk-fill flowable resin-based composite base materials. J Dent 2012; 40: 500-505.

23- Sertac Peker, Figen Eren Giray, Basak Durmus, Nural Bekiroglu, Betül Kargül \& Mutlu Özcan Microleakage in class $\mathrm{V}$ cavities prepared using conventional method versus Er:YAG laser restored with glass ionomer cement or resin composite. J Dent 2016; 509-519.

24- Ferracane JL, Mitchem JC. Relationship between composite contraction stress and leakage in class V cavities. Am. J. Dent. 2003; 16: 239-243.

25- De Moor RJ, Delme KI. Laser-assisted cavity preparation and adhesion to erbium-lased tooth structure: part2. Present-day adhesion to erbium-lased tooth structure in permanent teeth.J.Adhes. Dent. 2010;12:91-102.

26- Delmé KI, Deman PJ, De Bruyne MA, et al. Microleakage of four different restorative glass ionomer formulations in class V cavities:Er:YAG laser vs .conventional preparation. Photomed Laser. Surg.2008;26:541-549.

27- Sarah S. Al-Angari, Anderson T. Hara, et al. Physicomechanical properties of a zinc-reinforced glass ionomer restorative material. Journal of Oral Science, 2014; 56:(1)11-16.
28- Van Noort, R.. Introduction to Dental Materials 2nd edition. London Mosby. 2002p.236.

29- Scherrer, S. S. and W. G. de Rijk .. The fracture resistance of allceramic crowns on supporting structures with different elastic moduli. International Journal of Prosthodontics 1993; 65:462-467.

30- Lee, S. K. and P. R. Wilson . Fracture strength of all-ceramic crowns with varying core elastic moduli. Australian Dental Journal, 2000; 45( 2):103-107.

31- Farah, J. W. , J. A. Hood , and R. G. Craig. Effect of cement bases on the stresses in amalgam restorations. J Dent Res.1975;54(1):10-5.

32- Traini, T., Sinjari, B., Pascetta, R., Serafini, N., Perfetti, G. Trisi, P., Caputi, S.,. The zirconia-reinforced lithium silicate ceramic: lights and shadows of a new material. Dent Mater. 2016;35 (5): 748-755.

33- Gupta R., Parkash, H., Shah, N., \& Jain, V. Aspectrophotometric evaluation of color changes of varioustooth colored veneering materials after exposure to common lyconsumed beverages. The Journal of Indian Prosthodontic Society. 2005; 5(2): 72-78.

34- Marwa Tannir, Essam Osman, Mohammad Rayyan, And Ehab A. Farghaly. Fracture Resistance And Color Stability Of Two Hybrid Ceramic Veneers Versus Lithium Disilicate Glass Ceramic Veneers. Egyptian Dental Journal 2018; 64: 1-5.

35- Willard A, Gabriel Chu TM. The science and application of IPS e.Max dental ceramic. The Kaohsiung Journal of Medical Sciences 2018;34 (4): 238-242.

36- Preis V, Behr M, Hahnel S, Rosentritt M. Influence of cementation on in vitro performance, marginal adaptation and fracture resistance of CAD/CAM-fabricated ZLS molar crowns. Dent Mater. 2015;31(11):1363-1369.

37-Franz S, Stefan S R and Schmitter M. Fracture resistance of glazed, full-contour ZLS incisor crowns .Journal of prosthodontic research. 2017;61(3):344-349.

38-Sieper K, Wille S, Kern M. Fracture strength of lithium disilicate crowns compared to polymer-infiltrated ceramicnetwork and zirconia reinforced lithium silicate crowns. J Mech Behav Biomed Mater. 2017; 74:342-348. 